\title{
Artelogie
}

Recherche sur les arts, le patrimoine et la littérature de l'Amérique latine

$15 \mid 2020$

Latin American networks: Synchronicities, Contacts and Divergences.

\section{Diego Rivera et Élie Faure : Contributions du peintre à la critique française des arts du Mexique ancien}

María Isabel Quintana Marín

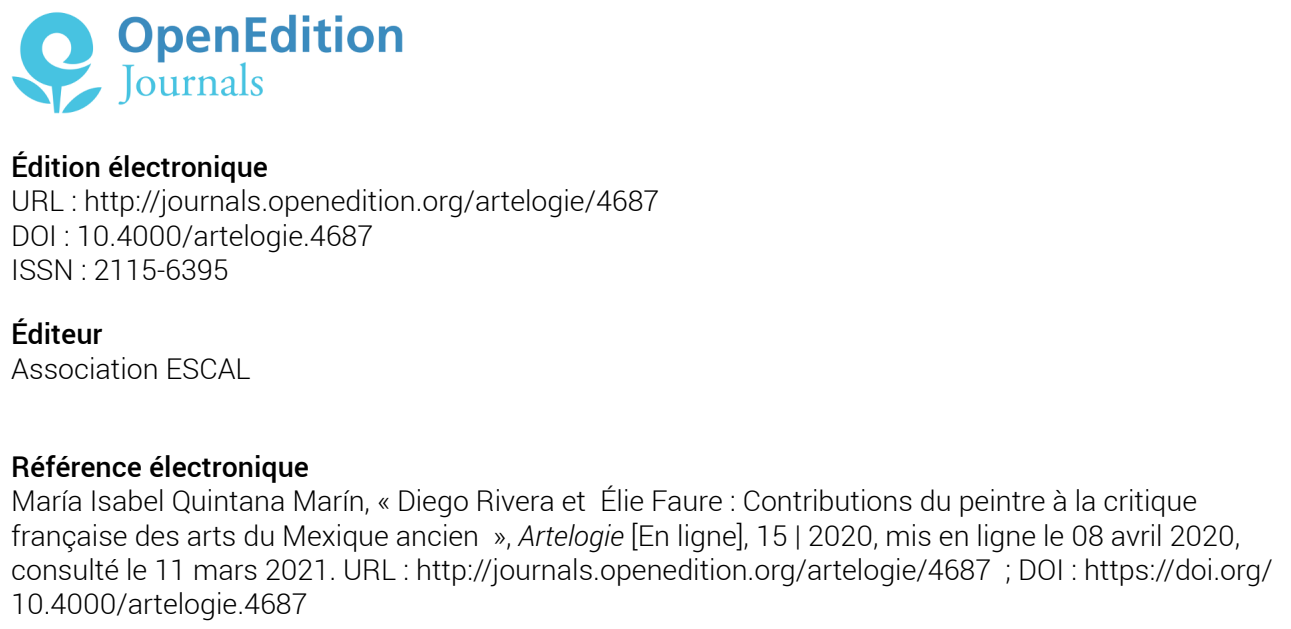

Ce document a été généré automatiquement le 11 mars 2021.

Association ESCAL 


\title{
Diego Rivera et Élie Faure : Contributions du peintre à la critique française des arts du Mexique ancien
}

\author{
María Isabel Quintana Marín
}

\section{Introduction}

1 En 1918, vers la fin de la Grande Guerre, Diego Rivera et Élie Faure se rencontrent. À cette époque, le peintre mexicain a déjà rompu avec le cubisme. Entre 1913 et 1917, il avait fait des tableaux cubistes après s'être intéressé pour un temps au néoimpressionnisme, puis étudié le Greco et Cézanne lors d'une période précubiste. Cependant, de sérieuses confrontations théoriques avec ses confrères s'étaient soldées par un changement de voie le menant de retour à la figuration.

2 Cette rencontre est marquante tant pour le peintre mexicain que pour l'historien de l'art français. Rentré au Mexique en 1921, Diego Rivera reconnaît en Élie Faure l'un de ses «maîtres»1. En effet, il y a dans son approche artistique des échos d'élie Faure au sujet de l'ordre social, de la fonction de l'art, de l'architecture en tant que manifestation de l'âme collective et lieu d'intégration populaire et artistique ${ }^{2}$. Élie Faure, lui, se dit redevable à Rivera d'une découverte du Mexique où il se rend en 1931 afin d'étudier les civilisations précortésiennes ${ }^{3}$. «Si je suis venu au Mexique c'est grâce à Diego qui m'a initié il y a quinze ans dans mes sympathies pour le Mexique », déclare$\mathrm{t}-\mathrm{il}^{4}$.

3 Pourtant, avant leurs échanges, Élie Faure s'était déjà intéressé à ce pays ; il avait publié des pages sur ses arts anciens en s'attardant sur les Aztèques dans le deuxième volume de son Histoire de l'art consacré à l'art médiéval en $1911^{5}$. Pourquoi parler donc d'une initiation auprès de Rivera, et ignorer cette publication témoignant d'un certain nombre de recherches effectuées, de certaines connaissances acquises et transmises sur 
l'histoire et les arts du Mexique ancien? Cette omission volontaire soulève des questions à propos d'un revirement critique concernant ses anciennes recherches, d'une découverte particulière du passé artistique mexicain issue de sa rencontre avec Rivera dont les premières appréciations écrites sur l'art précortésien - tout au moins connues au moment de la rédaction du présent article - datent de 1916. En quoi, quand et dans quelles circonstances Rivera lui aurait-il apporté en ce domaine un nouveau regard?

4 Des éléments de réponse à cette question seront abordés par une approche évolutive de la période comprise entre 1911 - année de publication de l'Histoire de l'art : l'art médiéval, mais aussi de l'installation de Rivera à Paris - et 1931 quand Élie Faure fait ses déclarations au Mexique. Tout d'abord, l'analyse des premiers textes d'élie Faure et de Diego Rivera sur l'art précortésien s'impose. L'intention étant d'identifier des sources et d'observer en quoi leurs positions se différencient ou se rapprochent avant leur rencontre. Ensuite, leur période d'échanges en France sera traitée afin de déterminer dans quel contexte ils ont lieu, à quel type de discours de Rivera sur l'art précortésien Faure a-t-il pu se trouver exposé et avec quelle intensité, et à quel degré le critique d'art a pu les adopter à cette époque. Enfin, il sera possible de suivre la progression de la pensée de Faure entre 1921 et 1931, tout en identifiant de possibles consonances théoriques avec le peintre, des moments d'échanges ou de transmission.

\section{Deux visions des arts du Mexique ancien}

Élie Faure écrit quelques pages sur l'art mexicain dans son Histoire de l'art : l'art médiéval de 1911. N'étant jamais allé au Mexique, il se sert de sources scientifiques pour se documenter. Pour son texte, il fait recours à l'archéologue et homme de lettres Auguste Génin, domicilié au Mexique. Faure lui demande des informations et lit ses Poèmes Aztèques écrits entre 1884 et 1889 et publiés en 1890. Les illustrations proviennent en grand nombre de Briquet, certainement Alfred Briquet, photographe à Mexico ${ }^{6}$. Il utilise aussi des clichés de la maison Giraudon et de C.-B. White, sans doute Charles Betts Waite, photographe états-unien actif au Mexique avant la Révolution.

6 Auguste Génin écrit en poésie l'histoire des Aztèques en quatre parties - les Légendes, les Mexis, la Conquête, les Ruines -, tout en soulignant la base scientifique de son travail ${ }^{7}$. Pour préparer à la lecture de ses poèmes, l'auteur retrace en prose l'histoire des Aztèques dans son avant-propos. Il remonte aux origines du peuplement du continent en supposant l'entrée par l'Amérique du Nord de vagues migratoires successives qui retrouvent des races autochtones, primitives ${ }^{8}$. Descendues vers le Sud, les différentes migrations qui se déroulent au cours des siècles - entre autres, les Otomis, les Mayas, les Zapotèques, les Toltèques - laissent des traces architecturales qui "attestent l'existence d'une civilisation préhistorique originale et parvenue à une haute perfection $»^{9}$. Avec les Chichimèques et les Aztèques, héritiers des Toltèques, "commencent les temps historiques $»^{10}$. Bien qu'il décrive les Aztèques comme ayant " un caractère ombrageux et jaloux; ambitieux et braves à l'excès " ${ }^{11}$, il affirme que "leur cruauté a été considérablement exagérée par certains historiens " ${ }^{12}$. Génin s'interroge avec Prescott sur « quel était le plus sauvage du vainqueur et du vaincu " $^{13}$, et il adhère aux affirmations de Bartolomé de las Casas qui entend certains récits effrayants à propos des Aztèques comme des « évaluations de brigands " colonisateurs cherchant à justifier leurs propres actes de barbarie ${ }^{14}$. Les Aztèques «n'étaient pas des 
barbares ", affirme Génin. Ils avaient une religion « sanguinaire » et étaient cruels dans la guerre, "mais ils avaient fait pourtant de notables progrès dans la voie de la civilisation $»^{15}$. Il admire leur vie domestique, leur législation, leur amour de la poésie, leur peinture, leur sculpture ; « ils étaient parvenus à un incroyable degré de perfection dans les arts mécaniques et surtout dans la science astronomique ", écrit-il ${ }^{16}$.

Dans Histoire de l'art: l'art médiéval, Élie Faure se rapproche de Génin dans certains aspects, mais il s'en éloigne à propos de l'art. Faure mentionne un possible déplacement vers le territoire mexicain de peuples conquérants - Toltèques, Chichimèques, Aztèques -, entrés dans le continent probablement par l'Amérique du $\operatorname{Nord}^{17}$. Ces peuples rencontrent des Inöits ${ }^{18}$ et des Indiens nomades, groupements étudiés par Élie Reclus dans Les Primitifs. Les flux se succèdent et la rencontre des nouveaux arrivés avec ceux qui les ont précédés crée des synthèses artistiques. Cependant, ces peuples conquérants vivaient un "trouble ardent». Connaisseurs de l'astronomie et de la nature, adorateurs du soleil en tant que générateur de vie, ils devaient pourtant couvrir les murs des temples de sang humain ${ }^{19}$. Contrairement à Auguste Génin, Élie Faure souligne la sauvagerie des Aztèques qui héritent des Toltèques « l'art, le culte du soleil, la soif du sang ", et dont les dieux ont besoin de « cadavres frais $»^{20}$. Ainsi, en décrivant l'environnement à Tenochtitlan, il se figure des "flots de sang ", " des amas de têtes coupées ", « une horrible buée rouge $»^{21}$.

Cette perception des Aztèques détermine la lecture qu'Élie Faure effectue de leur art en se demandant :

«Du fond de cette horrible buée rouge qui montait de partout, prenait à la gorge, faisait rouler dans les veines un poison nauséeux, voilait le souvenir, comment l'âme énervée et découragée des peuples eût-elle pu saisir ou même entrevoir un possible équilibre et dégager des formes qui l'environnaient ces grandes lois de la structure vivante d'où sortit, par l'Égypte et la Grèce, la civilisation? $»^{22}$.

Pour Élie Faure, l'Égypte ancienne et la Grèce antique, tout comme le Moyen-âge français qui dresse des cathédrales, représentent de grandes époques d'association et de rayonnement artistique. L'unité spirituelle qui leur est propre suscite la recherche des structures dans l'art et l'éclosion d'une architecture reflétant l'ordre social. L'art de ces civilisations exprime la synthèse, l'harmonie et l'équilibre, l'unité de leurs sociétés. Au Moyen-âge, le peintre, le tailleur de pierre, les guildes d'artisans travaillant au cœur de la cathédrale française cherchent ces qualités et expriment leur esprit constructif en ce qu'ils ont à réaliser. L'Inde et la Chine connaissent aussi cette splendeur artistique. Ce n'est visiblement pas le cas à Tenochtitlan où les bas-reliefs des murailles, si richement décorés qu'ils pouvaient l'être, «disparaissaient sous le sang "; où "une vapeur d'abattoir masquait les idoles"; où "la tradition de la matière sculptée ne pouvait se transmettre à des générations mutilées ${ }^{23}$. Leur sculpture laisse percevoir un certain souci de "symétrie essentielle», dit Faure, mais "non seulement ils ne pouvaient pas dépasser, mais ils ne pouvaient pas atteindre l'étape architecturale de l'évolution de l'esprit ${ }^{24}$. Ainsi écrit-il :

«Ce n'est qu'une vague apparence qui a fait comparer les idoles de pierre que leurs outils de bronze dégageaient peu à peu du bloc aux purs colosses égyptiens dont les plans se répondent, s'amènent l'un l'autre et se balancent comme le flot des sables et des mers. $»^{25}$

10 Le critique d'art explique davantage la cause de cet art inabouti des Aztèques en raison de «leur destin épouvantable [qui] les avertit qu'ils n'auront pas le temps d'étudier le sens de la forme, de s'élever dans l'abstraction, de parvenir à la notion d'harmonie. $»^{26}$ 
Cette conscience d'un si court avenir face au panorama terrifiant laissé par les sacrifices, se traduit sur le plan formel :

« En hâte, ils disent ce qu'ils ont à dire, des visions confuses et violentes, brèves, morcelées, un cauchemar pesant de tristesse et de cruauté. Même quand ils élèvent des statues entières, quand ils abandonnent pour un jour leurs combinaisons hiéroglyphiques de figures géométriques et de formes animées, on dirait à leur façon d'articuler les membres et d'architecturer les masses, qu'ils n'ont jamais vu que des troncs mutilés, des membres épars, des crânes scalpés, des faces écorchées aux orbites vides, où claque le rictus des dents. La vie n'est là que par tronçons, coupée comme elle est dans leur âme, n'ayant que des tressaillements courts, figée par le dogme et la peur. ${ }^{27}$

11 L'historien de l'art affirme que tous les morceaux de corps vus, les Aztèques les "combinent en formes confuses, vaguement architecturées » dans leurs statues ${ }^{28}$. Il illustre la quête d'une certaine "symétrie essentielle» en décrivant une sculpture représentant un dieu " accroupi » sur un « socle ornementé », " les yeux caves au ciel », qu'il identifie à Tlaloc. Il s'agit en réalité de Xochipilli, ces lignes mettant en évidence des imprécisions quant à la connaissance d'Élie Faure de la mythologie aztèque. Sous la reproduction photographique de l'idole, la légende identifie également la sculpture au « dieu de l'eau ». Il reste à savoir si cette information erronée ne lui a pas été transmise par le fournisseur du cliché, Alfred Briquet.

12 Élie Faure avait publié un extrait de son Histoire de l'art : l'Art médiéval dans la revue L'Art et les artistes ${ }^{29}$ que le Journal amusant commentait de cette manière : «Élie Faure, dont on connaît le talent de philosophe d'Art, nous entretient des prodigieuses et effrayantes conceptions esthétiques des Aztèques. ${ }^{30}$ C'est l'impression que laisse ce texte nourri de récits sur la sauvagerie des Aztèques et d'informations obtenues par des intermédiaires qui font le pont entre le Mexique et le critique d'art qui n'y a jamais mis les pieds.

13 Cette année de 1911, Diego Rivera s'installe à Paris. Il est difficile de savoir si le peintre a l'occasion de feuilleter ce numéro de l'Art et les artistes, tout comme il est difficile de savoir s'il lit l'Histoire de l'art : l'Art médiéval dès sa publication. Il n'y a pas pour l'instant de trace connue attestant de quelque intérêt particulier de Rivera pour l'art préhispanique à cette époque-là. En revanche, dans les années qui suivent, Diego Rivera rejoint les peintres cubistes qui, eux, s'intéressent aux arts nègres et aux grandes civilisations comme l'Égypte, ces modèles artistiques atteignant pour eux la «quatrième dimension » qu'ils désirent exprimer en peinture. Guillaume Apollinaire explique cette quête des peintres nouveaux. Ils cherchent certes la géométrie qui est « aux arts plastiques ce que la grammaire est à l'art de l'écrivain ${ }^{31}$. Cependant, au-delà de la géométrie euclidienne, ils souhaitent exprimer de «nouvelles mesures possibles de l'étendu » : c'est la " quatrième dimension » ${ }^{32}$. Celle-ci est la "dimension de l'infini », une « mesure de la perfection » qui donne aux objets « les proportions qu'ils méritent dans l'œuvre ${ }^{33}$. Cette mesure, dit-il, « permet à l'artiste peintre de donner à l'objet des proportions conformes au degré de plasticité où il souhaite l'amener ${ }^{34}$. Pour Apollinaire, les artistes apportant aux hommes une idée sublime de l'univers, les jeunes peintres «s'éloignent de plus en plus de l'ancien art des illusions d'optique et des proportions locales pour exprimer la grandeur des formes métaphysiques ${ }^{35}$. C'est ainsi qu'Apollinaire associe le cubisme au " grand art », à " l'Art religieux ${ }^{36}$. Picasso, Braque, Metzinger, Gleizes, Marie Laurencin et Juan Gris, dit Apollinaire, éliminent l'« accident visuel et anecdotique » pour exprimer la "réalité essentielle [...] rendue avec une grande pureté $»^{37}$. Cézanne, ayant exploré la « réalité profonde », est placé en 
précurseur de ce procédé par Gleizes et Metzinger dans Du "Cubisme $»^{38}$. Apollinaire écrit que cette démarche explique la prédilection des peintres nouveaux pour certaines expressions artistiques : "[...] cette imagination : la quatrième dimension, n'a été que la manifestation des aspirations, des inquiétudes d'un grand nombre de jeunes artistes regardant les sculptures égyptiennes, nègres et océaniennes, méditant les ouvrages de science, attendant un art sublime $[. ..] »^{39}$.

Or, Rivera transpose ces principes aux arts anciens de son pays, comme le prouve un texte adressé en 1916 à Marius de Zayas. Ce dernier expose quelques-unes de ses œuvres dans sa galerie newyorkaise cette année-là. Après quelques lignes sur le traitement cézannien de la forme en toute indépendance de l' "accident perçu ", Rivera fait écho aux idées d'Apollinaire: "Dans l'espace plastique les choses ont une dimension supra-physique, qui s'accroît ou diminue en relation directe avec l'importance que son existence a dans l'esprit du peintre ${ }^{40}$. Il cite comme exemple la perspective hiérarchique des primitifs italiens. Il prône la pureté de la forme, de la couleur et de la matière, la «forme pure » comme offrant « une vision spirituelle de l'univers». Il affirme que le peintre doit confronter «l'existence des choses dans l'espace réel, visuel et physique ; et leur existence dans l'espace réel, supra-physique et spirituel $\aleph^{41}$, la forme, la couleur et la matière devant être traitées indépendamment de l'accident visuel, en respectant leur pureté. Un tel traitement a lieu, dit-il, « dans les peintures de Giotto, de Cézanne, du Greco, de Zurbarán, de Velázquez ou dans l'art oriental et surtout, dans la sculpture mexicaine et nègre $»^{42}$.

Diego Rivera affichait un certain intérêt pour intégrer dans son œuvre les apports esthétiques de son pays avant même que la guerre n'éclate, quand des lignes colorées dans ses tableaux s'inspiraient du sarape. Puis, lors de son séjour en Espagne entre 1914 et 1915, il a été sans doute affirmé dans sa démarche. À Madrid, le Mexique en pleine révolution a dû être au cœur de ses entretiens avec Alfonso Reyes, Ángel Zárraga, Jesús T. Acevedo et Martín Luis Guzmán. C'est en 1915 que voit le jour La Querella de México, un opuscule où Guzmán dénonce "l'absence du sentiment et de l'idée de Patrie » chez les dirigeants et les partis mexicains ${ }^{43}$. Il estime nécessaire une éducation d'ordre moral, spirituel, afin que le pays parvienne à résoudre « le problème de son existence normale en tant que peuple organisé $\aleph^{44}$. Cette même année, Reyes rédige Visión de Anáhuac (1519). Dans ce récit qui ne nie pas l'existence à Tenochtitlan des "têtes de mort exposées » et des " témoins abominables des sacrifices" qui font «fuir le soldat chrétien $»^{45}$, Reyes privilégie le moment où les Conquistadors découvrent les Aztèques menant une vie harmonieuse au milieu d'une nature généreuse ; un peuple aux mœurs raffinés, organisé en guildes d'artisans, créatif, aimant la poésie et les fleurs, riche en moyens artistiques et avec une importante architecture publique: des temples, des marchés et des palais ${ }^{46}$. Il dépeint une ville structurée, conçue géométralement, où « les édifices se regroupent en masses cubiques $»^{47}$, et $"[$ les] rues rayonnants [prolongent] les arêtes de la pyramide $»^{48}$. Reyes ne condamne pas pour autant la rencontre avec l'Europe d'un regard nostalgique, il ne prétend pas à des « perpétuations absurdes de la tradition indigène ", pas plus qu'à des "perpétuations de la [tradition] espagnole »; mais il invoque « la communauté de l'effort pour dominer [la] nature sauvage et rude ", ainsi que la « communauté, beaucoup plus profonde, de l'émotion devant le même objet naturel », qui relient les Mexicains à la race du passét9. Il faut, suggère-t-il dans son récit, «l'œuvre de l'action commune » et « l'œuvre de la contemplation commune » ${ }^{50}$. 
16 Il n'est pas étonnant que Rivera, après avoir nourri son âme mexicaine dans cet environnement, rentre à Paris plus qu'engagé dans une sorte de "mexicanisme » pictural. Il introduit encore des éléments allusifs au Mexique dans son Portrait de Martín Luis Guzmán et surtout dans El Guerrillero o Paisaje zapatista, choix qui choque la grande majorité de ses pairs dérangés par son "exotisme " ${ }^{51}$. Seul Picasso aurait salué sa démarche au départ, faisant changer favorablement les avis de la plupart d'entre eux ${ }^{52}$. Bien des années après, Rivera affirme avoir peint El Guerrillero dans un «état d'esprit mexicain » et poursuivi de façon intuitive la «tradition de l'art mexicain d'avant la conquête » dans ses tableaux de l'époque ${ }^{53}$. Il dit également avoir développé pleinement son « exotisme » ou « coefficient mexicain » en $1916^{54}$.

17 Ainsi, avant leur rencontre, Élie Faure et Diego Rivera construisent des visions très différentes sur l'art précortésien. Faure, qui analyse surtout l'art des Aztèques, confirme à travers ses observations la sauvagerie rattachée à ce peuple et une détresse morale. Rivera pour sa part, idéalise l'ancienne sculpture mexicaine en l'associant aux paradigmes artistiques des peintres cubistes.

\section{Diego Rivera et le Mexique dans le milieu artistique et intellectuel parisien}

18 Nous pouvons désormais nous attarder sur la circulation des idées de Rivera dans le milieu parisien et les possibles appropriations pour la part d'Élie Faure entre 1918 et 1921. Il est judicieux néanmoins de revenir un peu en arrière pour voir quelle place occupe le Mexicain dans la vie parisienne et quel genre de raisonnement il partage.

19 Au moment de sa rencontre avec Élie Faure, Diego Rivera était déjà une personnalité marquante dans le milieu intellectuel et artistique où il s'était donné de faire découvrir son pays. Le peintre, qu'Ilya Ehrenbourg dit avoir rencontré en 1913, se faisait remarquer à La Rotonde pour sa taille imposante, mais aussi car «il agitait un bâton mexicain sculpté $\|^{55}$. «Il était de ces gens qui n'entrent pas dans une pièce, mais la remplissent tout de suite de leur présence ", se souvient Ehrenbourg ${ }^{56}$. Son bâton sculpté a dû sans doute suscité des conversations sur le Mexique avec les nombreux habitués du café ch7 $^{5}$. Le poète russe rapporte la nature de leurs conversations : « Diego me parlait du Mexique, et moi je lui parlais de la Russie " ${ }^{58}$. Il offre des détails sur le contenu des causeries de Rivera :

«Diego aimait parler du Mexique, de son enfance. Il vivait depuis dix ans à Paris, [...], mais il avait toujours devant les yeux les montagnes rousses couvertes de cactus, les paysans coiffés de larges chapeaux de paille, les mines d'or de Guanajuato, les révolutions incessantes : Madero renverse Dias [sic], Huerta reverse Madero, les partisans de Zapata et de Villa renversent Huerta.

En écoutant Diego, je me mis à aimer le mystérieux Mexique; les sculptures des Aztèques se fondaient avec les partisans de Zapata $"^{59}$.

Ilya Ehrenbourg transcrit les idées d'une conversation qu'il date de début 1917, entamée un soir à La Rotonde avec Rivera, Boris Savinkov, Max Volochine, le modèle Margot et Modigliani, et qui se poursuit chez ce dernier avec en plus Fernand Léger et Pavel Lapinski ${ }^{60}$. La chronologie d'Ehrenbourg est douteuse quant à la présence de Max Volochine et de Fernand Léger à Paris début 1917 ; l'année 1916 serait plus probable. Retenons néanmoins les idées échangées lors de cette discussion. Ils causaient «sur la guerre, sur l'avenir, sur l'art». Léger pensait à une reconstruction des territoires 
détruits qui solliciterait des ouvriers, des ingénieurs, des techniciens ; l'art s'inspirerait alors du travail, de la technique, du sport et de la science. C'était un avenir devant lequel Ehrenbourg ne se montrait pas optimiste : «Je déteste les machines », disait-il. Léger croyait à un réveil populaire, idée que Lapinski confirmait en supposant un proche dénouement dans lequel les socialistes pourraient intervenir réagissant contre le capitalisme destructeur. Pour Léger, l'art devrait suivre ce mouvement populaire. « Il faut une approche nouvelle » affirmait-il, «l'Art survivra s'il devine le langage de notre époque $\aleph^{61}$. Le moment était arrivé pour que Rivera intervienne. Il confirmait la décadence de l'art à Paris et prenait parti pour un art du peuple et pour le peuple, tout en soulevant la sensibilité artistique des révolutionnaires mexicains et l'exemple d'action collective laissé par les Aztèques :

«Paris meurt, l'art meurt, disait Rivera. Les paysans de Zapata n'ont jamais vu aucune machine, mais ils sont cent fois plus modernes que Poincaré. Je suis persuadé que si on leur montre notre peinture, ils comprendront. Qui a construit les cathédrales gothiques ou les temples aztèques? Tout le monde. Et pour tout le monde. $»^{62}$

Rivera mettait en relief ensuite l'apport salutaire de l'art des "barbares » et des "sauvages » à l'art actuel. "L'art doit avaler une gorgée de barbarie, affirmait-il. La sculpture nègre a sauvé Picasso. Vous irez tous bientôt au Congo ou au Pérou. Il vous faut passer par l'école de la sauvagerie $»^{63}$. Le Mexicain éclairait Ehrenbourg au sujet de son désespoir face à l'avenir dépeint par Léger : « Ilya, tu es pessimiste, parce que tu es trop civilisé. [...]. Tu es Européen, c'est là ton malheur. L'Europe agonise. Les Américains, les Asiatiques, les Africains vont venir... $»^{64}$

Ehrenbourg dresse le portrait d'un Rivera engagé dans une valorisation de son pays, convaincu, d'une part, de la sensibilité esthétique des Aztèques et des populations paysannes du Mexique actuel, et, d'autre part, du potentiel révolutionnaire, tant politique qu'esthétique, de ces populations. Rivera fait ressortir chez les Aztèques un mode de fonctionnement collectif qui donne lieu à des réalisations à dimension sociale comme le temple. Puis, il met en relief les facultés esthétiques des révolutionnaires de Zapata qui les rendent capables de comprendre la peinture cubiste. Reliant le présent au passé, il inclut les Aztèques et les paysans de Zapata dans cette "école de la sauvagerie » qu'il faut suivre, les associant à l'art nègre qui a "sauvé Picasso ». C'est cette " gorgée de barbarie » qu'il croit nécessaire à l'art. Ehrenbourg présente Rivera en train de renverser des jugements de valeur; de souligner les facultés du monde extraeuropéen à donner des leçons en matière d'art et de civilisation; de mettre en exergue des qualités sociales et esthétiques chez des peuples soi-disant « non civilisés » car non européens.

C'est en 1918 que Diego Rivera situe sa rencontre avec Élie Faure ${ }^{65}$ et c'est à cette date qu'ils apparaissent en contact. Rivera peint le portrait de Faure en tenue militaire rentré du front, il n'est pas encore démobilisé ; ce dernier collabore avec l'exposition de Rivera et d'autres peintres retournés à la figuration qui se tient chez Eugène Blot entre octobre et novembre. Quelques lignes de l'essai d'Élie Faure sur Paul Cézanne sont citées dans le catalogue à manière d'avant-propos ${ }^{66}$. Rivera, bien qu'ayant quitté le cubisme, se revendique de Cézanne, de l'instinct qui conduit à la recherche des structures, ce qu'Élie Faure admire et défend chez le Maître d'Aix qui annonce, selon lui, une nouvelle génération d'artistes bâtisseurs au cœur d'un nouvel ordre social. Faure s'entend très bien avec celui qu'il surnomme l' " Aztèque ${ }^{67}$. En 1933, il rappelle à Rivera combien sa rencontre a été décisive : 
«Nous avons une communion de pensée que je pense complète. C'est une grande joie pour moi. Tu ne sauras jamais quel événement capital fut cette rencontre avec toi il y a douze ans. Tu étais le poète du Nouveau Monde surgissant soudainement devant mes yeux depuis l'inconnu. J'ai ressenti en te connaissant et en te comprenant une sensation inouïe de délivrance... $»^{68}$

Bien que la chronologie d'Élie Faure s'avère imprécise quant à l'année où ils font connaissance, cet extrait témoigne de l'empathie commune qu'ils éprouvent. Avant d'entrer en contact, le peintre mexicain et le critique d'art français ont déjà des idées politiques qui les rapprochent et des visions artistiques qu'ils y associent. En effet, leurs manières de considérer la dimension sociale de l'art et de concevoir l'architecture de création et à usage collectifs s'apparentent. Par surcroît, Ehrenbourg présente Rivera en train d'évoquer l'exemple de la cathédrale dans le contexte d'une conversation qui pressentit l'avènement d'un mouvement populaire socialiste changeant l'ordre social et artistique. Or, Élie Faure conçoit la Commune et la cathédrale comme des modèles pour l'instauration d'un nouvel ordre social collectif fédéré par les organisations corporatives et syndicales. Pour Faure, la cathédrale est issue d'une émancipation populaire. Ainsi, ils ont tous les deux des conceptions politiquement révolutionnaires et visionnaires du passé médiéval ${ }^{69}$.

Cependant, auprès de Diego Rivera, Élie Faure entend parler du Mexique comme jamais auparavant: "Il me contait sur le Mexique, où il était né, des choses extravagantes. Mythologue, me disais-je, peut-être mythomane », se souvient-il ${ }^{70}$. En ce qui concerne la passé artistique mexicain, Faure se retrouve face à des opinions différentes à la sienne. Tout d'abord, à cette compréhension du passé aztèque à l'image du passé médiéval; à cette mise en parallèle entre le temple aztèque et la cathédrale pour démontrer la puissance collective. Quand Faure affirme qu' "il n'y a pas d'architecture monumentale sans cohésion sociale » et qu'il voit l'art actuel «[obéir] à un obscur besoin de subordination à quelque tâche collective ", Rivera se dit que c'est « ce qu' [il

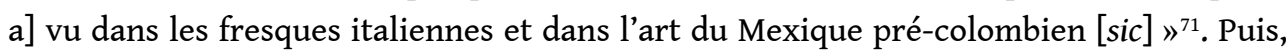
Faure apprend d'autres lectures esthétiques sur l'art précortésien. À l'époque de leurs échanges parisiens, Rivera se trouve très engagé dans ses interprétations modernes de l'art préhispanique, comme en témoigne David Alfaro Siqueiros. Ce dernier arrive en Europe en 1919, après avoir participé à la Révolution dans les files de l'armée constitutionaliste à côté des paysans, des ouvriers, des indiens du Mexique. Siqueiros raconte qu'au contact du peuple, des tempéraments, de la géographie, de l'archéologie et de l'art du Mexique, de ses arts populaires et de toutes ses manifestations culturelles, lui et d'autres artistes avaient compris que "l'art avait eu un grand rôle social dans toutes les périodes importantes de l'histoire $\aleph^{72}$. C'est imprégné de cette pensée qu'il affirme être parti pour l'Europe et avoir rejoint Rivera :

«Ainsi se fit la rencontre entre la nouvelle ferveur et les nouvelles idées des jeunes peintres mexicains qui avaient participé directement aux luttes armées de la révolution mexicaine, représentées par moi, et une période extrêmement importante de la révolution formelle dans les arts plastiques d'Europe, représentée par Rivera. $»^{73}$

En mai 1921, Siqueiros publie à Barcelone le fruit de leurs échanges dans « 3 llamamientos de orientación actual a los pintores y escultores de la nueva generación americana ", article paru dans le seul numéro de la revue Vida americana. Dans ce texte inspiré des principes cézanniens, il invite les artistes à réaliser un art universel dans un "esprit constructif» qui vise l'équilibre et les formes essentielles, «la structure géométrale» obtenue par les "grandes masses primaires: cubes, cônes, sphères, 
cylindres, pyramides » qui composent $«$ le squelette de toute architecture plastique $»^{74}$. À ces fins, il convoque à une observation du passé précolombien :

«La compréhension de l'admirable fond humain de l' "art nègre", et de l'art "primitif " en général a donné claire et profonde orientation aux arts plastiques [...] ; rapprochons-nous pour notre part, des œuvres des anciens habitants de nos vallées les peintres et sculpteurs indiens (MAYAS, AZTĖQUES, INCAS, etc., etc.); [...]. . ${ }^{75}$

Siqueiros préconise l'adoption de leur "énergie synthétique» et de la "vigueur constructive de leurs œuvres» qui relèvent d'une observation de la nature ${ }^{76}$. Cette inspiration du passé doit dialoguer avec un regard sur le présent, sur les villes modernes et les productions techniques qui suscitent des "émotions plastiques $»^{77}$. Il reconnaît ce manifeste comme étant une combinaison des idées rapportées par lui du Mexique et de celles de Rivera correspondant au «mouvement rénovateur du Paris d'alors $»^{78}$.

Faisant échos à certaines de ces conceptions, Élie Faure parvient à souligner des valeurs esthétiques dans l'art aztèque, bien que moins catégoriquement. Ses propos de 1911 se trouvent nuancés, voire révisés, dans son édition augmentée de l'Histoire de l'art : l'art médiéval de 1921 où il modifie certaines phrases et rajoute des paragraphes. Tout d'abord, il reconnaît une certaine présence de lois dans l'art aztèque, quoique pas « tout à fait » saisies. Il écrit alors :

« Du fond de cette horrible buée rouge qui montait de partout, prenait à la gorge, faisait rouler dans les veines un poison nauséeux, voilait le souvenir, comment l'âme énervée et découragée des peuples eût-elle pu tout à fait dégager des formes qui l'environnaient ces grandes lois de la structure vivante d'où sortit, par l'Égypte et la Grèce, la civilisation de l'Occident ? $»^{79}$

Puis, le critique d'art trouve un critère de comparaison avec les œuvres de l'Égypte: "C'est par l'intuition de la masse, non par l'intelligence du profil qu'on peut comparer les idoles de pierre que leurs outils de bronze dégageaient peu à peu du bloc aux purs colosses égyptiens [...]», écrit-il ${ }^{80}$. Il ne s'agit plus d'une "vague apparence" trompeuse comme en 1911, mais d'une « intuition de la masse » qui rend possible cette comparaison. En outre, il rajoute quelques lignes sur l'aspect structurel et l'équilibre obtenus grâce au « souci d'une symétrie essentielle » chez les Aztèques : «Sans doute parviennent-ils souvent ainsi, par un effort qu'on sent douloureux vers l'expression la plus tranchante, à des résumés structuraux profondément émouvants, un équilibre soudain qui arrête et assied la forme titubante avec l'énergie du désespoir même. ${ }^{81} \mathrm{Ce}$ n'est qu'au "premier abord", dit-il désormais, que "têtes et tronçons de reptiles, crânes dénudés, doigts humains, bréchets d'oiseaux", tous ces morceaux qui composent la sculpture, "semblent accrochés au hasard", car il y a bien une "continuité du monstre composite", différente certes de celle des figures égyptiennes ${ }^{82}$. En réalité, écrit-il, «[...] une architecture sommaire, mais imposante, faisant masse sous toutes ses épaisseurs, et vue par l'ensemble vivant plus que par le plan abstrait, le ramène à l'unité organique sans qui l'œuvre s'effondrerait. $~^{83}$ Enfin, ce n'est plus, comme écrivait-il en 1911, « qu'ils [n'ont] pas le temps d'étudier le sens de la forme ", mais " qu'ils [n'ont] pas le temps d'en approfondir le sens " ${ }^{84}$; et leurs statues, où ils « combinent en formes confuses des morceaux d'animaux vivants", ne sont plus "vaguement architecturées", ces deux derniers mots étant supprimés de la phrase dans la nouvelle édition. 
30 Mais Élie Faure mentionne aussi des aspects sociaux des Aztèques en concordance avec les affirmations de Rivera. Alors que dans l'Histoire de l'art de 1921 Faure aborde des particularités esthétiques et expressives, dans L'Arbre d'Eden, recueil d'articles imprimé en août $1922^{85}$, il inclut le passé mexicain dans son répertoire des grandes époques d'association. Faure décèle chez ses contemporains, pourtant très individualistes, une «connaissance approfondie de tous ces rythmes collectifs - égyptianisme [sic], chaldéisme, hellénisme, primitif, brahmanisme, bouddhisme, christianisme, islamisme, mexicanisme - où l'individu n'apparaissait pas $»^{86}$. Ce sont des époques d'une «forte synthèse religieuse », dit-il, dont l'attrait ne fait qu'annoncer l'avènement d'un nouveau rythme collectif ${ }^{87}$.

31 Élie Faure n'est pas le seul à se laisser séduire par le passé précortésien. En 1921, l'art du Mexique ancien se trouve légitimé à Paris en tant que source d'enseignements pour l'art moderne à l'instar des arts nègres et des œuvres des civilisations anciennes. Albert Gleizes fait référence à la fascination que cet ensemble de modèles exerce sur les artistes. "Les grands monuments asiatiques, égyptiens, mexicains, voire les arts plastiques nègres, conseillent les jeunes artistes tandis qu'ils ne s'intéressent guère à la Grèce et à la Rome ", dit-il début 1921 à l'occasion de l'exposition internationale de Genève ${ }^{88}$, à laquelle Diego Rivera participe avec des travaux de différents périodes. Gleizes explique la supériorité des époques autres que la gréco-romaine, parmi lesquels il inclut le Mexique d'avant les Aztèques: «Les arts asiatiques, égyptiens, toltèques, musulmans se développent dans un sens d'absolu spirituel, ceux de la Grèce dans un absolu purement matériel $»^{89}$; alors que les premiers ont " des proportions au collectif dont l'âme unique est devenue sensible", les seconds procèdent d'une "dimension à l'individu en hypnose sur lui-même $\|^{90}$. Sur le rapport des premiers à la réalité, Gleizes explique :

«L'élément réaliste n'est que le départ d'une évolution vivante, aboutissant à créer le type, suscitée par l'idée religieuse chez les asiatiques et les égyptiens. Chez les anciens mexicains [sic] et les musulmans cet élément réaliste est tellement réduit à sa vertu d'essence, que l'idée est incorporée à des formes pures, géométriques, régies par des quantités mobiles mathématiques. $»^{91}$

32 Ainsi, le regard moderne sur des sociétés et des arts anciens du Mexique parvient à se fixer dans les mentalités au lendemain de la Grande Guerre. Sans aucun doute, Diego Rivera part après avoir joué un rôle majeur dans la construction de ce regard. Élie Faure, parmi d'autres, se trouve influencé par ses conceptions jusqu'à un certain degré. Mais sa vision continue à évoluer après le départ du peintre.

\section{Élie Faure sur les valeurs universelles des arts précortésiens}

Les écrits et les échanges d'Élie Faure avec le critique d'art états-unien Walter Pach permettent de suivre sa pensée des années 1920. Walter Pach, ami de longue date et traducteur de ses ouvrages, étudie l'art précortésien lors d'un temps passé à Mexico en 1922. Dès lors, des échanges à propos du Mexique et de Rivera se mettent en place entre le critique d'art français et le critique d'art nord-américain. Ce dernier, qui avait découvert l'œuvre de Rivera en 1916 dans la galerie de Marius de Zayas ${ }^{92}$, assiste au réveil artistique qui privilégie la peinture murale et s'inspire du passé préhispanique. C'est alors qu'il fait la connaissance de Rivera, Jean Charlot et d'autres peintres. 
Dès son arrivée à Mexico en 1921, Rivera avait déclaré vouloir étudier l'art populaire et les ruines préhispaniques ${ }^{93}$. Il avait confirmé et diffusé auprès des artistes mexicains les propos du texte publié par Siqueiros dans Vida americana. "Ce que l'artiste européen cherche avec tant d'empressement, ici, au Mexique, se trouve manifesté surtout dans l'art national abondamment $"^{94}$, affirmait-il convaincu que les artistes pourraient tirer un grand profit de l'étude de l'art maya, aztèque et toltèque ${ }^{95}$. Au Mexique, se trouvait "la plus merveilleuse architecture" et "la sculpture ancienne la plus pure et solidement plastique au monde», une sculpture «d'ensemble» et "en bloc, par excellence ", écrivait-il pour Azulejos ${ }^{96}$. Contrairement au caractère décomposé que Faure accordait à l'ancien art aztèque en 1911, pour Rivera, si quelqu'un avait dépecé le corps humain, c'était bien Rodin. Il reprochait aux artistes mexicains d'avoir suivi l'exemple du sculpteur français par la représentation "d'étranges dépouilles humaines", d'" hommes sans tête, qui se tordaient ", de "morceaux de torse qui marchaient sur des cuisses amputées $»^{97}$. Il invitait les artistes à ne pas "dépecer le corps humain ", mais à rechercher les formes essentielles : «le cylindre, le cône, la sphère ", sous l'inspiration des monuments et des arts précortésiens ${ }^{98}$. C'est dans cette atmosphère à la fois traditionaliste et avant-gardiste que Pach rencontre Rivera en 1922, année du retour de Siqueiros.

Cette même année, le musée d'ethnographie à Paris se réaménage pour exhiber des pièces d'art précortésien et des objets actuels d'intérêt ethnographique en provenance du Mexique dont Auguste Génin fait don. Élie Faure n'a pas dû manquer de suivre avec beaucoup d'intérêt cet évènement, et surtout, les avis critiques de René Verneau, directeur du musée. L'anthropologue est surtout favorable à l'art ancien, présentant les Aztèques comme ayant une civilisation plus développée que les Toltèques, avec des monuments comparables à ceux de l'Égypte et du Annam ${ }^{99}$. Chez les zapotèques - deux statuettes zapotèques illustrent la dernière page de l'article -, Verneau souligne tout particulièrement "un sens de l'effet décoratif extrêmement original» et une "stylisation des formes toute spéciale, très monumentale ${ }^{100}$. C'est l'occasion pour Guillaume Janneau d'évoquer dans le Bulletin de la vie artistique la "Renaissance» mexicaine qui est en train de s'accomplir en expliquant: "Quelques artistes, retournant aux sources d'inspiration des ancêtres, - faut-il nommer Angel Zarraga ? en retrouvent les grands accents $\aleph^{101}$. Selon Janneau, ils mériteraient d'être exposés aussi au Trocadéro ${ }^{102}$. De quoi nourrir le goût grandissant d'Élie Faure pour le passé mexicain. Alors que la réorganisation du musée fait du bruit à Paris, Faure écrit à Walter Pach qu'il a « une admiration très grande et chaque jour accrue » pour le " vieil art mexicain». Avec des qualificatifs qui évoquent les opinions de Verneau, Faure rajoute: «C'est l'un des plus stylisés qui soient, et des plus nettement originaux. Je ne regrette qu'une chose : c'est de ne pas lui avoir donné plus de place dans mon livre. Ce sera peut-être pour une autre édition $»^{103}$. Il doit se rapporter à son Histoire de l'art : l'art médiéval où il s'attarde sur des aspects esthétiques et que Pach traduit cette année-là.

En 1926, L'Amour de l'art publie «L'Art au Mexique. I. Les Musées», un article de Walter Pach sur l'art du Mexique ancien tout imprégné des observations esthétiques de Rivera. À propos des anciennes créations précortésiennes, l'auteur avertit qu' « il faut du temps pour en apprécier la gradeur $\aleph^{104}$, pour se rendre compte qu'au-delà de leur aspect "effrayant", ces œuvres s'inscrivent dans un cadre universel ${ }^{105}$. Comme chez les Chaldéens et les Égyptiens, il décèle dans une sculpture de Chalchiuhtlicue une conscience de l'ordre cosmique qui a déterminé « ses grandes masses », « ces angles » 
et « ces plans formidables ${ }^{106}$. Cette conscience, écrit-il, permet que «la chose vue et l'abstraction " coexistent dans l'œuvre. Puis, dans deux serpents sculptés, il identifie "des calculs mathématiques, astronomiques", le nombre d'écailles correspondant à des chiffres en rapport avec l'univers ${ }^{107}$.

«On a écrit tant de pages sur le côté d'horreur dans l'art mexicain, écrit Pach, qu'il me semble utile de donner encore des exemples du génie évolué, équilibré, de cette race. Si on les accepte, ou plutôt si on reconsidère nos illustrations et pénètre leur beauté, on sentira le côté défectueux de notre point de vue lorsqu'il est appliqué mécaniquement à un peuple dont les conceptions de la vie, du temps et de l'espace sont si différentes des nôtres. $»^{108}$ formes paru en 1927, mais s'il mentionne à nouveau les représentations de morceaux de corps chez les Aztèques, c'est en justifiant cette caractéristique autrement que par la sauvagerie : «Les statues aztèques qui saignent, tronçons coupés, monstres composites où des gueules en sang, des mains pourries, de crocs et des griffes se mêlent, ne font qu'accentuer l'expression d'attente anxieuse du dieu Tlaloc, espérant la pluie comme un pauvre homme dont le soleil a calciné le champ. $»^{113}$ L'esthétique à l'image de la boucherie provoquée par des dieux assoiffés de sang dans l'Histoire de l'Art: l'Art médiéval de $1911^{114}$ devient celle qui traduit l'état d'âme d'un dieu assoiffé d'eau dans L'Esprit des formes. Et ce dieu est à l'image des hommes. En réalité, derrière la représentation de Tlaloc, Faure décèle un drame humain et commun à un peuple qui attend la pluie en temps de sécheresse.

Mais au-delà des particularités expressives, Faure souligne un aspect qui relie l'art du passé mexicain à celui d'autres civilisations. En Chine, aux Indes, en Égypte, l'image d'un dieu taillé par une multitude d'hommes, "semblait jaillir d'un même cœur, être conçue par une même tête, réalisée par une même main "; ce fut le cas aussi en France avec « la cathédrale aux mille voix » et au Mexique : «En Chine, aux Indes, comme au Mexique ou en Islam, comme en Égypte, comme dans la Grèce dorienne et l'Europe médiévale, le héros est presque inconnu. $»^{115}$ Le Mexique ancien se trouve bien parmi 
les périodes aux "civilisations grandioses $»^{116}$. Pour Faure, «l'esprit des formes est un " ${ }^{117}$, où que l'on aille, malgré la diversité stylistique permettant de distinguer une civilisation d'une autre. Cet esprit correspond à une quête universelle commune qui donne lieu à des " analogies surprenantes de structure, de rythme, d'accent ", assurant à l'œuvre sa stabilité essentielle et l'expression de lois durables ${ }^{118}$.

«Si l'on conçoit cela, souligne-t-il, les formes les plus éloignées des apparences de la vie, - l'art aztèque, par exemple, qui est à peu près illisible au premier coup d'œil et réunit, dans ses équilibres de masses, les objets et les organes les plus hétéroclites et souvent les moins définis, - deviennent immédiatement et pleinement intelligibles ${ }^{119}$.

Il n'y a pas pour l'instant d'évidence matérielle - pour le moins connue au moment de l'écriture de cet article - prouvant l'existence d'échanges entre Élie Faure et Diego Rivera sur l'art préhispanique au cours des années 1920. Ils restent tout de même en contact à cette période-là et prévoient à plusieurs reprises de se revoir avant 1927. Devenu célèbre à Mexico grâce à Rivera en 1921, Faure affirme avoir reçu une invitation du Ministère des affaires étrangères pour s'y rendre à l'occasion du Centenaire de l'Indépendance, mais ce voyage ne se concrétise pas pour des raisons financières. Invitation, mais pas d'argent. "Rivera aurait dû y veiller », se confie-t-il déçu à Charles Péquin ${ }^{120}$. Une nouvelle tentative de retrouvailles est prévue pour la fin 1924, comme l'écrit Guadalupe Marín à Faure en octobre $1923^{121}$. Octobre 1926, Élie Faure informe Walter Pach d'une nouvelle invitation reçue ${ }^{122}$, mais ce projet n'aboutit pas non plus. Ils ne se revoient qu'en 1927 quand Rivera, après avoir vu Pach à New York ${ }^{123}$, passe une journée avec Élie Faure avant de se rendre à Moscou pour les célébrations du dixième anniversaire de la Révolution d'octobre ${ }^{124}$. Les causeries en tête à tête sur L'Esprit des formes n'ont pas dû manquer. Il leur faudra attendre presque quatre ans pour se retrouver à nouveau, cette fois-ci au Mexique, où Faure se rend enfin à l'été 1931 lors de son périple autour du monde. Son but premier, dit-il, est d'étudier les anciennes civilisations locales ${ }^{125}$. En compagnie de Rivera, le visitant français se trouve immergé dans la société mexicaine et visite des lieux emblématiques du pays, bien qu'il rencontre aussi d'autres artistes, de même que l'archéologue Porfirio Aguirre qui lui apporte certainement des connaissances. C'est à Aguirre que Faure dédicace son article « Réflexions sur l'art mexicain » qui parait dans L'Illustration fin 1932. Il aurait prévu de retourner au Mexique en hiver pour visiter les ruines mayas ${ }^{126}$, mais ce retour n'a pas lieu.

Élie Faure déclare avoir sur ce pays des impressions complexes, mais vis-à-vis de l'art aztèque, ses idées sont claires dans une lettre à sa fille : «[...] j'admire sans réserve l'ancien art aztèque. C'est peut-être plus beau que l'Égyptien. $»^{127}$ Faure remarque que les Aztèques sont ceux qui «[ont infligé] à la civilisation et à l'art précortésiens leur caractère classique $»^{128}$. Concernant leur sculpture, il écrit : «Leur art présente sans doute, au premier abord, un caractère atroce, qui répond à ce que nous révèle un examen superficiel des mœurs du Mexique précolombien. $»^{129}$ Il avertit ainsi de la nécessité d'étudier le passé mexicain en profondeur pour saisir la valeur esthétique de cet art. Après une observation de leur organisation administrative et sociale, de leurs conceptions cosmogoniques et de leur connaissance de l'univers, Faure souligne le sens de l'ordre, l'éthique et le progrès scientifique de cette société où «la mystique, la connaissance et l'art étaient d'accord». Il rajoute que "le sens ésotérique de cet art doit, par conséquent, se chercher sous son apparence effroyable ${ }^{130}$. Certes, du point de vue expressif, l'art des Aztèques traduit un "goût des supplices ", mais il exprime aussi 
une douce sensibilité. Faure relève cet aspect tout en avouant ses lectures erronées de 1911 :

«À l'analyse pourtant, l'horreur de cet art s'atténue, en tout cas s'explique. Je dois faire ici amende honorable, n'ayant su moi-même y voir, jadis, que la cruauté du prêtre et le sadisme du bourreau. Il montre bien souvent une humanité, parfois même une tendresse d'autant plus émouvantes qu'elles se combinent, dans la même harmonie plastique, à son apparence globale de férocité. ${ }^{131}$

Enfin, Élie Faure consacre la «métaphysique grandiose » de cet art en l'associant à l'art moderne:

"On a beaucoup parlé, depuis vingt ans, des "équivalences plastiques", ce qui a provoqué chez nous ce mouvement d'un intérêt philosophique si particulier qui a traversé le cubisme entre Picasso et Braque et laisse après lui l'ébauche d'une ère architecturale nouvelle. L'art aztèque, en ceci, nous a précédés de loin. Il a réalisé des synthèses spirituelles que notre théologie anthropomorphique et notre morale dualiste ne nous autorisaient pas même à entrevoir. ${ }^{132}$

À l'exception de quelques "études » et de certains serpents en pierre, «tout est en équivalence plastique ici", écrit-il ${ }^{133}$. Il salue le sens de l'unité des Aztèques qui combinent dans la même masse des objets qui n'ont entre eux aucun rapport naturel de continuité, ni même de contigüité, et moins encore de parenté anatomique. $»^{134}$ Il les conçoit comme les héritiers de la discipline architecturale des Toltèques et met en exergue leur esprit constructif qui se lie à l'emploi du symbole, à l'invention d'un "monde suprasensible ", à la transposition des lois universelles ${ }^{135}$. Ils ont exprimé en "synthèses de pierre " les lois de la nature et les désirs de leurs dieux ${ }^{136}$. "Royaume de la pierre, comme seule l'Égypte, ailleurs ", écrit-il137. Il admire également le rythme, la géométrie, «la représentation réelle, l'équivalence, l'abstraction incorporées au symbolisme universel [qui] s'offrent d'elles-mêmes au statuaire [...] $»^{138}$. Sans mentionner la " quatrième dimension », il affirme tout de même que l'art aztèque "c'est une combinaison sans fin de tous les angles de vue » et qu'il suggère " une dimension complémentaire » qui correspondrait à une " conquête par le temps des dimensions de l'espace " $^{139}$. Faure ne peut que conclure: "Ici vécut et mourut la culture la plus originale de l'Histoire, avec celle de l'Égypte, de la Chine et de l'Inde des Védas. $»^{140}$

L'étude de l'art ancien mexicain permet à Faure de saisir aussi le mysticisme collectif des Toltèques qui l'éclaire davantage sur d'autres moments de cohésion religieuse dans l'histoire de l'humanité :

« Non seulement la religion cosmique des vieux Toltèques, la plus "scientifique" de toutes, me fut ainsi révélée dans sa forte cohésion, mais aussi beaucoup d'autres qui n'ont rien à voir avec elle, l'Hellénisme, l'Indouisme, le Catholicisme, l'Islamisme, le constructions freudiennes des peuples primitifs, nègres et polynésiens au premier rang, qui impriment à leurs groupements arbitraires la vraisemblance d'un édifice spirituel organiquement conçu. $»^{141}$

\section{Conclusion}

Ainsi, les premiers textes de Faure et de Rivera révèlent qu'avant leur rencontre, ils acquièrent des visions très différentes des arts du Mexique ancien. Pour Élie Faure, les sculptures aztèques composées de tronçons de corps ne font qu'exprimer la détresse d'un peuple troublé par la pratique des sacrifices. Leur aspect fragmentaire empêche que des valeurs esthétiques universelles propres aux arts des civilisations à grande 
cohésion religieuse comme l'Égypte ancienne ou la Chine se manifestent. Ses observations confirment l'image véhiculée par certains récits historiques sur la sauvagerie aztèque. Diego Rivera, lui, a une lecture de la sculpture précortésienne en accord avec la compréhension que les peintres cubistes ont des arts d'Afrique, d'Océanie et de l'Égypte ancienne comme traduisant des réalités métaphysiques et universelles. Il suit les traces de Cézanne et des théoriciens du cubisme - Gleizes, Metzinger et Apollinaire - et manifeste ainsi un mexicanisme teint de modernité européenne.

Rivera partage ses réflexions sur le passé précortésien avec son entourage parisien. Elles sont à portée tant esthétique que sociale, présentant les Aztèques comme un peuple uni dans un même effort, créateur d'œuvres à fonction sacrée et collective. À l'époque de ses échanges parisiens avec Élie Faure, le peintre, toujours inspiré des leçons de Cézanne, se trouve en pleine réflexion avec Siqueiros sur l'esprit constructif des civilisations préhispaniques et les valeurs universelles de leurs arts. C'est alors que Faure fait preuve d'un regard en cours de transformation à l'égard de l'art des Aztèques où il identifie, bien que modestement, des proximités esthétiques avec celui des grandes civilisations qu'il admire : une intuition des masses, un certain équilibre, une démarche architecturale, un sens de l'unité. En outre, il inclut le passé mexicain parmi les époques ayant connu la cohésion religieuse et sociale.

Après le retour de Rivera au Mexique, Faure continue à bâtir sa vision de l'art aztèque tout en s'intéressant à ses valeurs universelles. S'il semble adapter l'avis scientifique d'un René Verneau en termes de style et d'originalité, ses lectures du sens expressif se modifient aussi en accordant à l'art aztèque une grande dose d'humanité au-delà de son aspect terrifiant. Puis, il reconnaît dans ses structures l'expression d'une âme collective. Il nourrit son approche grâce à une circulation transnationale et transatlantique des idées modernes sur l'art précortésien, qui connecte le Mexique, les États-Unis et la France. Lors de son voyage au Mexique en 1931, ses analyses formelles le mènent à mettre en relief chez les Aztèques une forme de création par équivalences plastiques qui les place en prédécesseurs des peintres cubistes. Ses observations s'étendent aussi vers d'autres civilisations comme les Toltèques, chez qui il reconnaît une grande tradition architecturale et une foi collective à même de l'éclairer sur d'autres civilisations à forte cohésion religieuse.

51 Il a été dit qu'Élie Faure n'a pas été le seul à connaître un changement de regard à propos du Mexique ancien dans le milieu artistique parisien des années $1910^{142}$. L'étude du contexte de ses échanges avec Rivera a conduit à mettre en lumière la position d'Albert Gleizes au début des années 1920, bien qu'il reste à savoir dans quelle mesure il a pu l'adopter durant le temps passé aux États-Unis, puisque les idées avant-gardistes sur l'art précortésien ont traversé l'Atlantique pendant la Grande Guerre. Ce milieu reste à étudier davantage, non seulement quant aux Européens ayant expérimenté ce changement de regard, mais aussi quant à la contribution d'autres Mexicains à cette transformation. Bien que Rivera ait joué un rôle majeur dans la diffusion de la vision moderne des sociétés et des arts précortésiens, il faudrait s'interroger sur la part accomplie par des artistes présents à Paris durant cette décennie tels Gerardo Murillo, Alfredo Best Maugard, Ángel Zárraga ou encore Siqueiros; tout comme il faudrait le faire à propos d'un possible accompagnement par des personnalités diplomatiques comme Alfonso Reyes et Alberto Pani. Retracer ces itinéraires personnels vaudrait la 
peine, les années 1910 s'avérant un moment décisif dans l'évolution du regard sur les arts préhispaniques vers des conceptions esthétiques modernes.

\section{NOTES DE FIN}

1. Voir Diego Rivera, "Los "retablos" son por hoy la verdadera pintura mexicana ». Dans Id. Obras : 1. Textos de arte / éd. Xavier Moyssén, Mexico, El Colegio Nacional, 1996, t. 1, p. 35. Article paru pour la première fois dans Azulejos, janvier 1922, n 5, p. 22-26.

2. Cette transmission est le sujet de la thèse Du cubisme à d'autres cathédrales : Diego Rivera et l' 'Art social' d'Élie Faure, préparée par l'auteure de cet article sous la direction de Philippe Dagen à l'Université Paris 1 et soutenue le 26 novembre 2016.

3. «Élie Faure nos habla del arte mexicano - su espontaneidad - casi puedo decir que en México todo hombre es un artista - Una invitación de Diego Rivera », El Nacional, 28 juillet 1931, p.12:

«Especialmente mi venida a México se debe a mi deseo de visitar la ruinas de las civilizaciones autóctonas. »

4. Ibidem :

«Si yo vine a México fue por Diego quien me inició hace quince años en mis simpatías por México. »

5. L'ouvrage consulté à la Bibliothèque Forney à Paris comporte une double date : 1912 sur la couverture et 1911 sur la page de titre. Dans une lettre de 1911, Faure compte apporter le volume à Bourdelle. Selon une lettre à Maurice Reclus, la Commission d'achat des Beaux-arts l'examine vers janvier 1912. Martine Courtois et Jean-Paul Morel signalent une publication en novembre 1911 dans Élie Faure, Paris, Séguier, 1989, p. 286.

6. Élie Faure, Histoire de l'art : l'art médiéval, Paris, Éd. H. Floury, 1911, p. 161.

7. Auguste Génin, Poèmes aztèques, Paris, Fischbacher, 1890, p. 22-23. Génin mentionne des sources comme Cortés, Bernal Díaz del Castillo, Torquemada, Bernardino de Sahagún, Clavigero, Prescott, Humboldt, parmi d'autres.

8. Ibid., p. 6-7.

9. Ibid., p. 8.

10. Ibidem.

11. Ibid., p. 11.

12. Ibid., p. 20.

13. Ibid., p. 21. Il s'agit d'une citation tirée de William H. Prescott. Histoire de la conquête du Mexique avec un tableau préliminaire de l'ancienne civilisation mexicaine et la vie de Fernand Cortés / publiée en français par Amédée Pichot, Paris, librairie de Firmin Didot Frères, 1946, t. I, p. 82. Pour Prescott, la sauvagerie des Espagnols est d'autant plus condamnable puisque non seulement ils exterminent des êtres humains, mais aussi toute une civilisation par la destruction d'œuvres de l'esprit. En outre, lorsque Prescott décrit les coutumes rituelles et guerrières des Aztèques, il mentionne souvent des pratiques des Romains, des Grecs et de l'Inquisition qu'il estime parfois plus sauvages.

14. Ibid., p. 20.

15. Ibid., p. 19.

16. Ibid., p. 19-20.

17. Élie Faure, Histoire de l'art : l'art médiéval, op. cit., p. 158. 
18. Aujourd'hui, des Inuits.

19. Élie Faure, Histoire de l'art : l'art médiéval, op. cit., p. 161. Faure généralise quant à la pratique des sacrifices humains par les trois peuples cités. Par la suite, il se rapporte précisément aux Aztèques.

20. Ibid., p. 162.

21. Ibidem.

22. Ibidem.

23. Élie Faure, Histoire de l'art : l'art médiéval, op. cit., p. 162.

24. Ibid., p. 164.

25. Ibidem.

26. Ibidem.

27. Ibidem.

28. Ibidem.

29. Voir Élie Faure, « L'Art aztèque ", L’Art et les artistes, avril-septembre 1911, t. XIII, [n 73-78], p. 18-23.

30. "L'Art et les artistes : revue d'art ancien et moderne des deux mondes », Le Journal amusant : journal humoristique, 22 avril 1911, 64 ${ }^{\mathrm{e}}$ année, $\mathrm{n}^{\circ} 617$, p. 3.

31. Guillaume Apollinaire, Méditations esthétiques: les peintres cubistes, $2^{\mathrm{e}}$ éd., Paris, Eugène Figuière et Cie., 1913, p. [15].

32. Ibidem.

33. Ibid., p. 16.

34. Ibidem.

35. Ibid., p. [18]

36. Ibidem.

37. Ibid., p. 24-25.

38. Albert Gleizes, Jean Metzinger, Du «Cubisme», Paris, Hermann Éditeurs, 2012, p. 15-16. Ouvrage publié pour la première fois en 1912.

39. Guillaume Apollinaire, Méditations esthétiques..., op. cit, p. 16-17.

40. Diego Rivera. «Marius de Zayas ", dans Id., Obras. 3. Correspondencia / réunie et présentée par Esther Acevedo, Leticia Torres Carmona et Alicia Sánchez Mejorada, Mexico, El Colegio Nacional, 1999, p. 28 :

"En el espacio plástico las cosas tienen una dimensión suprafísica, que crece o disminuye en razón directa de la importancia que su existencia tenga en el espíritu del pintor.»

41. Ibidem :

"[...], el pintor comienza a partir de dos principios opuestos e indiscutibles: la existencia de las cosas en el espacio real, visual, físico; y su existencia en el espacio real, suprafísico y espiritual. »

42. Ibidem:

"La materia, la substancia también vive como accidente; calidad de la pintura per se; como estructura substancial independiente del accidente visual y de sus consecuencias plásticas, [...], como en las pinturas de Giotto, Cézanne, el Greco, Zurbarán, Velázquez o en el arte oriental y sobre todo, en la escultura mexicana y negra."

43. Martín Luis Guzmán, La Querella de México. A orillas del Hudson. Otras páginas, $2^{\mathrm{e}}$ éd., Mexico, Cia. General de Ediciones S.A.,1970, p. 12.

44. Ibid., p. 36

45. Alfonso Reyes, Visión de Anáhuac (1519), Madrid, Índice, 1923, p. 27.

46. Ibid., p. 25.

47. Ibid., p. 22 :

"Agrúpanse los edificios en masas cúbicas; [...]. »

48. Ibid., p. [20] : 
"A sus pies, en un espejismo de cristales, se extendía la pintoresca ciudad, emanada toda ella del templo, por manera que sus calles radiantes prolongaban las aristas de la pirámide. »

49. Ibid., p. 64 :

"Cualquiera que sea la doctrina histórica que se profese (y no soy de los que sueñan en perpetuaciones absurdas de la tradición indígena, y ni siquiera fío demasiado en perpetuaciones de la española), nos une con la raza de ayer, sin hablar de sangres, la comunidad de esfuerzo por domeñar nuestra naturaleza brava y fragosa; esfuerzo que es la base bruta de las historia. Nos une también la comunidad, mucho más profunda, de la emoción cotidiana ante el mismo objeto natural. »

50. Ibidem.

51. Diego Rivera, My Art, My Life: An Autobiography / collab. Gladys March, nouv. éd., New-York, Dover publications, Inc., 1991, p. 65.

52. Ibid., p. 65-66.

53. Ibid., p. 65.

54. Diego Rivera, «Datos autobiográficos », dans Id., Obras: 1. Textos de arte / textes rassemblés par Xavier Moyssén, Mexico, El Colegio Nacional, 1996, p. 90. Texte paru pour la première fois dans El Arquitecto, mars-avril 1926, série II, n VIII, p. 3.

55. Ilya Ehrenbourg, Les Gens, les années, la vie / traduit du russe par Michèle Kahn, Lyon, Parangon/Vs, 2008, p. 154.

56. Ibid., p. 203.

57. Ibid., p. 153. Ehrenbourg mentionne un grand nombre d'habitués de La Rotonde, bien qu'il n'indique pas une chronologie précise. Cette liste peut inclure des personnes ayant fréquenté le café dans les années 1910 ou dans les années 1920, sans pour autant avoir coïncidé. Parmi les personnes mentionnées : Chagall, Soutine, Kisling, Gottlieb, Dunikovski, Apollinaire, Max Jacob, Jean Cocteau, André Salmon, Léger, Lhote, Metzinger, Gleizes, Severini, Élie Faure, Picasso, Maria Blanchard, Gris, Foujita, Diego Rivera et Ángel Zárraga.

58. Ibid., p. 199.

59. Ibid., p. 197.

60. Ibid., p. 199-201.

61. Ibid., p. 201.

62. Ibidem.

63. Ibidem.

64. Ibidem.

65. Diego Rivera, « Datos autobiográficos », éd. cit., p. 91.

66. L'essai «Paul Cézanne » d'Élie Faure paraît pour la première fois dans Portraits d'hier, $1^{\text {er }}$ mai 1910, n²8, p. 99-126. Il est repris dans Les Constructeurs en 1914.

67. Élie Faure, "[Lettre] 216 » [À Charles Péquin le 28 juillet 1920], dans Id., Cuvres complètes / préf. Henry Miller, Paris, Jean-Jacques Pauvert, 1964, t. III, p. 1021.

68. Lettre d'élie Faure à Diego Rivera daté du 20 janvier 1933, citée dans Bertram D. Wolfe, La Vie fabuleuse de Diego Rivera / traduit de l'anglais par Régine Cavallaro, Paris, Séguier, 1994, p. 229. Titre original The Fabulous Life of Diego Rivera [1963].

69. La possibilité d'une lecture par Rivera de certains articles d'Élie Faure véhiculant ses idée comme «L'Art social », «La Cathédrale et la Commune » et «L'Art pour le peule » n'est pas à écarter.

70. Élie Faure, «Mon Périple : au Mexique où se heurtent deux civilisations », Le Petit parisien, 24 septembre 1931, 56 a année, $\mathrm{n}^{\circ} 19931, \mathrm{p} .1$.

71. Bertram D. Wolfe, La Vie fabuleuse de Diego Rivera, op. cit., p. 89. Wolfe se base sur des extraits de l'Histoire de l'art : l'art moderne d'Élie Faure pour reconstituer ses propos.

72. David Alfaro Siqueiros, L'Art et la révolution / textes choisis pas Raquel Tibol, sous la direction de Georges Fournial, Paris, Éditions sociales, 1973, p. 12-13.

73. Ibid., p. 13. 
74. David Alfaro Siqueiros, « 3 llamamientos de orientación actual a los pintores y escultores de la nueva generación americana », Vida americana: revista norte, centro y sudamericana de vanguardia, mai 1921, n 1, p. 3.

75. Ibidem :

"La comprensión del admirable fondo humano del "arte negro" y del arte "primitivo" en general dió clara y profunda orientación a las artes plásticas perdidas cuatro siglos atrás en una senda opaca de desacierto; acerquémonos por nuestra parte, a las obras de los antiguos pobladores de nuestros valles los pintores $y$ escultores indios (MAYAS, AZTECAS, INCAS, etc., etc.); [...]. »

76. Ibidem.

77. Ibid., p. 2.

78. David Alfaro Siqueiros, L'Art et la révolution, op. cit., p. 13.

79. Élie Faure, Histoire de l'Art : l'art médiéval / nouv. éd. augmentée, Paris, G. Crès et Cie., 1921, p. 183.

80. Ibid., p. 184.

81. Ibidem.

82. Ibidem.

83. Ibidem.

84. Ibidem.

85. Jean-Jacques Pauvert, éditeur des CEuvres complètes d'Élie Faure en 1964, signale que tous les articles de L'Arbre d'Eden avaient été publiés antérieurement, bien que la date d' " Architecture et individualisme » ne soit pas déterminée. Voir Élie Faure, CEuvres complètes / préf. Henry Miller, Paris, Jean-Jacques Pauvert, 1964, t. III, p. 1143.

86. Élie Faure, "Architecture et individualisme », dans Id., Euvres complètes / préf. Henry Miller, Paris, Jean-Jacques Pauvert, 1964, t. III, p. 296.

87. Ibidem.

88. Albert Gleizes, "Réhabilitation des arts plastiques", dans Id., Tradition et cubisme. Vers une conscience plastique : Articles et conférences 1912-1924, Éditions La Cible, Paris, 1927, p. 92. Conférence prononcée en lien avec l'exposition internationale de Genève le 22 janvier 1921.

89. Ibid., p. 81.

90. Ibidem.

91. Ibidem.

92. Voir Laurette E. McCarthy, Walter Pach (1883-1958) The Armory Show and the Untold Story of Modern Art in America, Pensylvania, The Pensylvania State University, 2011, p. 130.

93. «Entrevista con el pintor Diego Rivera », El Universal: el gran diario de México, 21 juillet 1921, p.

11.

94. Ibidem :

"Lo que el artista europeo busca con tanto afán, aquí en México se encuentra manifestado, sobre todo en el arte nacional, de una manera abundante."

95. Ibidem:

«Y no quiero fatigar a usted hablándole de todo lo que puede sacar de provecho un pintor, un escultor, un artista, en una palabra, si contempla, si analiza, si estudia el arte maya, el azteca, el tolteca, los que, en mi concepto, no tienen que envidiar a ninguno. "

96. Diego Rivera, «La exposición de la Escuela Nacional de Bellas Artes », Azulejos, octobre 1921, $\mathrm{n}^{\circ} 3$, p. 22 .

97. Ibidem:

"¿Por qué en la tierra en que hay la maravillosa arquitectura de Teotihuacán, Mitla, Chichén, y la escultura antigua más pura y sólidamente plástica del mundo, la exhibición que nos dan nuestros pintores actuales parece representar los efectos de un descarrilamiento? 
¿Por qué, aquí, donde hay escultura de conjunto y de bloque, por excelencia; aquí, donde hay pirámides, nuestros jóvenes obreros de la plástica tienen propensión a fabricar extraños despojos humanos?

Hombres sin cabeza, que se tuercen; pedazos de torso que marchan sobre muslos amputados... [...]. »

98. Ibid., p. 23.

99. René Verneau cité dans Guillaume Janneau, «Au Musée de l'ethnographie », Bulletin de la vie artistique, 15 octobre $1922,3^{\mathrm{e}}$ année, $\mathrm{n}^{\circ} 20$, p. 468.

100. Ibidem.

101. Ibid., p. 469.

102. Ibidem.

103. Élie Faure, «[Lettre] 263 » [À Walter Pach le 22 octobre 1922], dans Id., CEuvres complètes / préf. Henry Miller, Paris, Jean-Jacques Pauvert, 1964, t. III, p. 1037.

104. Walter Pach, «L »Art au Mexique. I. Les musées », L'Amour de l'art, septembre 1926, $7^{\mathrm{e}}$ année, $\mathrm{n}^{\circ}$ 9, p. 288.

105. Ibidem.

106. Ibidem.

107. Ibidem.

108. Ibidem.

109. Ibid., p. 290.

110. Ibid., p. 293-294.

111. Élie Faure, «[Lettre] 347 » [À Walter Pach le 25 octobre 1926], dans Id., Euvres complètes / préf. Henry Miller, Paris, Jean-Jacques Pauvert, 1964, t. III, p. 1062.

112. Ibid., p. 1062-1063.

113. Élie Faure, Histoire de l'art : l'esprit des formes, Paris, G. Crès, 1927, p. 184.

114. Élie Faure. Histoire de l'art : l'art médiéval [1911], op. cit., p. 162

115. Élie Faure, Histoire de l'art : l'esprit des formes, op. cit., p. 66.

116. Ibid., p. 421.

117. Ibid., p. v.

118. Ibid., p. v-vi.

119. Ibid., p. vi.

120. Élie Faure, "[Lettre] 243 » [À Charles Péquin le 2 septembre 1921], dans Id., CEuvres complètes / préf. Henry Miller, Paris, Jean-Jacques Pauvert, 1964, t. III, p. 1032.

121. Voir Martine Courtois, Jean-Paul Morel, Élie Faure, Paris, Séguier, 1989, p. 154.

122. Élie Faure, « [Lettre] 347 », éd. cit., p. 1062.

123. Voir Laurette E. McCarthy, Walter Pach (1883-1958)..., op. cit., p. 134.

124. Voir Élie Faure, "[Lettre] 366 " [À Walter Pach le 19 décembre 1927], dans Id., CEuvres complètes / préf. Henry Miller, Paris, Jean-Jacques Pauvert, 1964, t. III, p. 1070.

125. «Élie Faure nos habla del arte mexicano... », art. cit., p.12.

126. Ibidem.

127. Élie Faure, « [Lettre] 419 » [À sa fille le 8 août 1931], dans Id., CEuvres complètes / préf. Henry Miller, Paris, Jean-Jacques Pauvert, 1964, t. III, p. 1088.

128. Elie Faure, "Réflexions sur l'art mexicain », L'Illustration: journal universel, 31 décembre $1932, \mathrm{n}^{\circ} 4687$, p. 613.

129. Ibid., p. 614.

130. Ibidem.

131. Élie Faure, « Mon Périple » [1932], dans Id., CEuvres complètes / préf. Henry Miller, Paris, JeanJacques Pauvert, 1964, t. III, p. 561.

132. Elie Faure, « Réflexions sur l'art mexicain », art. cit., p. 615. 
133. Ibidem.

134. Élie Faure, « Mon périple », éd. cit., p. 562.

135. Ibidem.

136. Ibidem.

137. Élie Faure, «Mon Périple : Le Mexique », Le Petit parisien, 30 septembre 1931, $56^{\text {ème }}$ année, $n^{\circ}$ 19937, p. 4.

138. Élie Faure, « Mon périple », éd. cit., p. 562.

139. Ibidem.

140. Ibidem.

141. Élie Faure, Reflets dans le sillage, Paris, Jean Flory, 1938, p. 156.

142. Cette évolution s'inscrit dans un contexte plus large abordé par des auteurs comme Benjamin Keen dans The Aztec Image in Western Thought et Élodie Vaudry dans Les Arts précolombiens : transferts et métamorphoses de l'Amérique latine à la France, 1875 -1945.

\section{RÉSUMÉS}

En 1931, s'étant rendu au Mexique pour étudier les anciennes civilisations locales, Élie Faure se dit redevable à Rivera de son attrait pour le pays. Cet article aborde les contributions critiques du peintre à l'historien de l'art sur les arts précortésiens. Avant leur rencontre en 1918, Faure voit dans la sculpture des Aztèques l'expression de leur sauvagerie, alors que Rivera transpose à l'ancienne sculpture mexicaine les principes esthétiques de Cézanne et du cubisme. Durant leurs échanges parisiens, le regard de Faure commence à se transformer dans le sens des idées du peintre qui se rattache toujours au Maître d'Aix et identifie dans l'art précortésien des démarches artistiques collectives. Après le retour de Rivera au Mexique en 1921, la pensée de Faure continue à évoluer. Lorsqu'il retrouve l'artiste en 1931, il confirme les liens entre l'art aztèque et le cubisme et honore d'autres civilisations du Mexique ancien..

En 1931, mientras se halla en México para estudiar las antiguas civilizaciones locales, Élie Faure declara adeudarle a Rivera su atracción por el país. Este artículo aborda las contribuciones críticas del pintor al historiador del arte sobre las artes precortesianas. Antes de su encuentro en 1918, Faure ve en la escultura de los aztecas la expresión de su salvajismo, mientras que Rivera transpone a la antigua escultura mexicana los principios estéticos de Cézanne y del cubismo. Durante sus intercambios parisinos, la mirada de Faure empieza a transformarse en el sentido de las ideas del pintor que sigue apegándose al maestro de Aix e identifica enfoques colectivos en el arte precortesiano. Después del regreso de Rivera a México en 1921, su pensamiento sigue evolucionando. Cuando vuelve a encontrarse con el artista en 1931, Faure confirma los vínculos entre el arte azteca y el cubismo, y rinde honor a otras civilizaciones del México antiguo.

\section{INDEX}

Mots-clés : Diego Rivera, Élie Faure, Aztèques, cubisme, arts précortésiens, Mexique ancien, civilisations préhispaniques.

Palabras claves : Diego Rivera, Élie Faure, aztecas, cubismo, artes precortesianas, México antiguo, civilizaciones prehispánicas 